\title{
El papel de los Comités Pro-Estado en la creación del estado de Baja California*
}

\author{
Lawrence Douglas Taylor Hansen**
}

Resumen: El artículo trata del papel de los Comités Pro-Estado en la formación de Baja California como estado. Se analizan las raíces de la autonomía política en la península, que se remontan al Porfiriato, así como el impacto de la Revolución Mexicana sobre este proceso. Se examinan los diversos movimientos pro-esta do que surgieron de 1929 en adelante la composición de sus grupos de integrantes, sus objeti vos y contribuciones principa les en términos de dirigir el sentimiento popular hacia la rea lización de esta meta.

Abstract: The article deals with the role of the Pro-State Committees in the formation the state of Baja California. It analyzes the roots of political autonomy in the peninsula, which go back to the Porfiriato, as well as the impact of the Mexican Revolution on this process. It also examines the various pro-State movements which emerged from 1929 on, with regard to the composition of the member groups, their objectives and principal contributions in terms of directing popular sentiment towards the fulfilment of this goal.

\footnotetext{
* Una versión anterior de este trabajo fue presentada en el First Americas Program Workshop, organizado por el Pacific Institute for Women's Health,que tuvo lugar del 21 al 24 de febrero de 1996 en Los Angeles,California,EUA.

** Departamento de Estudios Culturales de El Colegio de la Frontera Norte-Tijua na. Se le puede enviar correspondencia a: Blvd. Abelardo L. Rodríguez 2925, Zona del Río, Tijuana, Baja California, C.P. 22320, tel: 01(66) 31-35-35, fax: 31-20-48, correo electrónico:Itaylor@ colef.mx
} 


\section{Introducción}

Si bien Baja California constituye uno de los estados más jóvenes de la república, la historia del proceso a través del cual se transformó en estado es, sin embargo, altamente significativa por lo que se revela de la formación de la región fronteriza norteña y de la nación en general, durante el periodo de consolidación posrevolucionaria.

Esta historia ha sido tratada tradicionalmente por los historiadores y escritores - tanto nacionales como regionales- casi como un hecho consumado. Según esta interpretación, cuando la entidad ya contaba con los criterios para ser incorporada a la federación con esta categoría, así se hizo. No se han tomado en consideración, sin embargo, las tendencias hacia la autonomía existentes entre la pobla ción de la entidad, que se remontaban hasta finales del siglo anterior, ni de los elementos importantes en este proceso de evolución política.

Uno de estos elementos fueron los diversos comités u organiza ciones que se establecieron a lo largo del periodo territorial de Baja California (1931-1952), con el propósito de dirigir o canalizar el movimiento en favor de la conversión de la parte septentrional de la península en estado.

Los Comités Pro-Estado, como llegaron a ser denominados, ejercieron un papel decisivo en términos de dirigir el sentimiento popular hacia esta meta, así como manifestar estos deseos frente a las sucesivas autoridades nacionales. También proporcionaron, finalmente, una base importante en términos de liderazgo, una vez que el gobierno nacional había tomado la decisión de otorgar a la región la categoría de estado, para la posterior construcción política de la nueva entidad.

\section{Los inicios del movimiento hacia la creación del estado}

Los orígenes del movimiento hacia la autonomía política en la Baja California se remontan hasta el Porfiriato (1876-1911). 
En 1882, con motivo de dotar al entonces Partido Norte con una organización pública para poder defender políticamente la línea divisoria con el estado de California, la cabecera de la región fue transferida del antiguo pueblo minero de Real del Castillo al puerto de Ensenada. Esta decisión se basó en la fácil comunicación del puerto con el extranjero y con la capital del país, así como en el hecho de que ya contaba con la Aduana Marítima y el Juzgado Territorial del Partido Norte. ${ }^{1}$ Los ranchos de la municipalidad se dividían en cinco comisarías - Real del Castillo, El Rosario, San Telmo, Santo Tomás y Tijuana- , cada una de las cuales contaba con un juez de paz y un policía (Ortiz, 1989:296; Walther, 1983:151-154).

En 1888, al considerar que era preciso ejercer una vigilancia más eficaz sobre la región fronteriza del noroeste, el gobierno federal convirtió los dos partidos en distritos, cada uno de los cuales dependería directamente de la federación con sus propias cabeceras ${ }^{2}$ Bajo el régimen del general Luis E. Torres (1888-1894), el primer jefe político y militar del Distrito Norte, las antiguas comisarías municipa les fueron convertidas en secciones municipales. Las más importantes de éstas eran las deTijuana y Tecate, establecidas el 2 de abril de 1888 ( Walther, 1984:7-9/ 1986:33, 91-97, 120-121). Las demás

1 José María Rangel, jefe político del Partido Norte, a Carlos Díez Gutiérrez, el Secretario de Gobernación, 22 de marzo de 1882, y Rangel a Jorge Ryerson, presidente municipal de Real del Castillo, 15 de mayo de 1882, Walther (1983:7),

2 Un factor principal de esta consideración fue la necesidad de mantener una vigilancia sobre las operaciones de la Compañía Internacional Mexicana de Terrenos, que había obtenido del gobierno de Díaz una concesión para colonizar una gran parte de la zona. Decreto del 14 de diciembre de 1887 del Congreso de la Unión relativo a la división del territorio de la Baja California en dos distritos políticos. Archivo General de la Nación (de aquí en adelante citado como AGN), Fondo:Archivo General de la Nación, caja 315; en Instituto de Investigaciones Históricas, Universidad Autónoma de Baja Ca lifornia (de aquí en adelante citado como IIH/ UABC), Fondo: Pablo Herrera Carrillo, ca ja 3, exp. 55. Véase también la propuesta de Torres de que el paralelo 28 grados fuera la línea de demarcación entre los dos distritos. AGN, "Gobernación", v. 276, sección 2a, exp. 1; en IIH/ UABC, caja 28, exp. 21; y AGN, "Archivo General de la Nación", caja 315; en IIH/ UABC, Fondo: Pablo Herrera Carrillo, caja 4, exp. 12. Es posible que hayan influido en la decisión del gobierno central las recomendaciones expresadas por el Col.Andrés L. Tapia, jefe político del territorio, de julio de 1877 a enero de 1878, en favor de elevar la categoría política de la entidad. Walther (1986:33, 91-97, 120-121). 
secciones estaban constituidas por el puerto de Ensenada, que seguía siendo la cabecera de la entidad, Real del Castillo, Santo Tomás, San Telmo y El Rosario. En cada uno de los distritos existía un juez de paz,quien, además de las responsabilidades de su propio cargo, fungía como agente de la autoridad política regional. Los distritos también contaban con destacamentos de gendarmes para mantener la seguridad y el órden público. ${ }^{3}$

El Ayuntamiento de Ensenada, dirigido por el presidente Luis Legaspy, estaba conformada esencialmente por los comerciantes del puerto. Cobraba los impuestos que la ley le autorizaba e intervenía en los asuntos tocantes a los servicios en general, especialmente en el caso de las obras públicas. La población civil participaba activa mente en las elecciones, que se llevaban a cabo anualmente, incluso con la intromisión en determinadas ocasiones del jefe político. ${ }^{4}$

Además de este desarrollo importante de la actividad política en la nueva cabecera del distrito, surgieron dos comunidades a lo largo de la frontera entre las Californias, que cobrarían considerable importancia en este siglo: Tijuana y Mexicali. La primera, establecida a mediados de junio de 1889, creció tanto durante su primera déca da de existencia que, en septiembre de 1901, fue elevada a la categoría de subprefectura. ${ }^{5}$ En 1903, el general Celso Vega, el segundo jefe político, elevó a la recién establecida comunidad de Mexicali a la categoría de sección municipal y, el 3 de agosto de 1905, en re-

3 Walther $(1986: 33,72,133)$. Se habían destacada algunas fuerzas de seguridad en la región desde finales de la década de 1870. Véase Ortiz (1989:71-82).

4 Uno de los jefes políticos que intervenían en la elección del presidente municipal y de los regidores fue Celso Vega, quien ejerció el cargo de 1903-1911.Véase Sama niego $(1994: 62,64)$.

5 Walther (1982:9/ 1986:133, 143-148). El 15 de junio de 1889 puede ser toma da como fecha de nacimiento deTijuana como ciudad, dado que fue entonces cuando el agrimensor Richard Orozco terminó el trazo del poblado para su futuro crecimiento. Una reproducción de este mapa se encuentra en Barrientes de la Torre (1983:3133). Ya en 1898, alrededor de 20 residentes deTijuana habían solicitado a las autorida des de Ensenada que la sección municipal deTijuana fuera erigida en municipalidad. Véase Aguirre (1975:124). 
conocimiento de su crecimiento continuo, fue convertida en subprefectura. 6

La lucha revolucionaria de 1910-1920 aceleró el desarrollo de las instituciones políticas del Distrito Norte La política del gobierno federal en torno a la "mexicanización" de la península y de su unión más estrecha con el resto de la república, volvió a ser apremiante en vista de la revuelta en la región dirigida por el jefe socia lista Ricardo Flores Magón, presidente del Partido Liberal Mexicano, durante la primera mitad de 1911. La revuelta llevada a cabo por fuerzas revolucionarias magonistas en el Distrito Norte de la península, del 29 de enero al 22 de junio de 1911, si bien terminó en una derrota, constituyó el acontecimiento más significativo en la historia de la región durante el siglo actual en términos de reforzar el sentido de identidad nacional entre la población local, que ya esta ba en proceso de formación desde muchas décadas atrás (Taylor, 1995:25-47).

Después de la derrota de los rebeldes, los gobiernos regionales y federales sucesivos adoptaron una serie de medidas con el fin de integrar el territorio más estrechamente con el resto de la nación. Estosintentos iniciales, empero, no dieron lugar a resultados tangibles, debido a las condiciones turbulentas que prevalecieron a lo largo de la década de la lucha revolucionaria. ${ }^{7}$

A lo largo de todo este periodo, el ayuntamiento ensenadense disputaba constantemente con el jefe político sobre lo que consideraba la coartación de sus prerrogativas y el acaparamiento del dinero de los impuestos. Algunos de sus miembros se unieron en un bloque de protesta para solicitar al gobierno federal el otorgamiento de ma yor autonomía al cabildo, la eliminación del jefe político como má

\footnotetext{
6 Celso Vega al Secretario de Gobernación, 23 de abril de 1904, y Ramón Corral, el vicepresidente de México, aVega, 27 de mayo de 1904, en AGN, Fondo:Gobernación, v. s/ n, sección 3a, 854-904(2), exp. 1; en IH/ UABC, caja 14, exp. 44; Vega al Secretario de Gobernación, 23 de abril de 1926. AGN, Fondo:Gobernación, v. \$/ n, Sección 3a 854904(2), exp. 1; en IIH/ UABC, caja 14, exp. 44.

${ }^{7}$ Informe del general Manuel Gordillo Escudero sobre comunicaciones marítimas, ferrocarriles, caminos carreteros, comunicación fluvial, telégrafos, teléfonos y obras portuarias, del Distrito Norte de Baja California, 30 de noviembre de 1911. AGN, Fondo: Revolución, caja 1, carpeta 1, s.n.; en IH/ UABC, exp. 40.
} 
xima autoridad en la región, y la celebración de elecciones libres. 8 Su poder decayó con el traslado de la cabecera del distrito a Mexica li a finales de 1914. A partir de esta fecha, Mexicali empezó a cobrar importancia a raíz de su crecimiento económico, su colindancia con la frontera internacional, así como por las mayores posibilidades que ofrecía para comunicarse con el resto del país por medio de la construcción de caminos y ferrocarriles. 9

Durante el periodo de consolidación política en el Distrito Norte bajo la jefatura del coronel Esteban Cantú Jiménez de 1915-1920, así como el de los gobiemos posrevolucionarios de la década de 1920, se crearon en la región ciertas bases para fomentar su desa rrollo en general, así como estrechar sus lazos con el resto de la República. Cantú, quien había sido nombrado gobernador y comandante militar del distrito por la Convención revolucionaria a finales de enero de 1915, fue reconocido posteriormente por Venustiano Carranza en este puesto después del triunfo de éste en la lucha de facciones 10

\footnotetext{
8 General Manuel Gordillo Escudero, jefe político del Distrito Norte, al presidente Francisco I. Madero y al Secretario de Gobernación, 22 de febrero de 1912. AGN, Fondo: Gobernación, v. 359, sección 3a, 1911 (38), exp. 4; en IIH/ UABC, caja 94, exp. 16. Acusaciones y denuncias por parte del Ayuntamiento en contra de Gordillo Escudero. AGN, Fondo:Gobernación, v. 917, sección s/ s, exp. 1/ 5; en IIH/ UABC, caja 46, exp. 11; y AGN, Fondo: Periodo Revolucionario, caja 30, exp. 6; en IIH/ UABC, caja 2, exp. 5; caja 54, exp. 89; en IIH/ UABC, caja 2, exp. 17; caja 65, exp. 59; en IIH/ UABC, caja 1, exp. 34;caja 97,exp. 37/ 2; en IIH/ UABC, caja 1, exp. 6; caja 97, exp. 46; en IIH/ UABC, caja 2, exp. 25; caja 109, exp. 6; en IIH/ UABC, caja 2, exp. 16;Denuncias de Miguel V. Gómez,jefe político durante el régimen del presidente Victoriano Huerta (1913-1914), en contra del Ayuntamiento de Ensenada, ante el Secretario de Gobernación,septiembre de 1913, en AGN, Fondo: Gobernación, v. 500, sección 1ra, exp. 32; en IIH/ UABC, caja 47, exp. 70; David Zárate, jefe político interino, al Secretario de Gobernación, agosto de 1914. AGN, Fondo:Gobernación, v. 501, sección 1ra. exp. 26; en IIH/ UABC, caja 49, exp. 25; Periódico Oficial de Distrito Norte de Baja California (de aquí en adelante citado como PODNBC), 20 de junio de 1914.

9 PODNBC (1914); Manuel Balarezo, el gobernador del Distrito Norte, al Secretario de Gobernación, 3 de marzo de 1921. AGN, Fondo: Dirección General de Gobierno, Serie B.2.51.89, caja 15, exp. 89; en IIH/ UABC, caja 4, exp. 43.

10 The Calexico Chronicle, (1914); The San Diego Union (1914); nombramiento de Esteban Cantú como comandante militar y jefe político del Distrito Norte, en PODNBC (1915); Cantú (1956:66-67); Dunn (1956:72-80).
} 
Durante su régimen, Mexicali se convirtió en el primer municipio de la zona con una población considerable. Cantú persuadió a Carranza para que se declararan caducas las concesiones que el gobierno de Porfirio Díaz había otorgado en 1888, a la Mexican Land and Colonization Company (Compañía Mexicana de Colonización y Terrenos), una empresa británica, y la Lower California Development Company (Compañía para el Desarrollo de Baja California), una compañía dependiente. Con la finalidad de crear una fuerza laboral para la naciente industria agrícola en el Valle de Mexicali, se invitaron a asentarse en el Valle de Mexicali a colonos chinos, japoneses, hindúes y mexicanos refugiados de la lucha intestina en el resto del país. ${ }^{11}$ El primer auge algodonero, junto con los ingresos provenientes de los permisos y cuotas cargados a las casas de prostitución, los casinos y otras fuentes de diversión enTijuana y Mexicali (cantinas, salas de billares, corridas de toros, el teatro, etcétera), permitieron la construcción de varias obras públicas, la más importante de las cuales fue el inicio del Camino Nacional. 12

Durante este periodo, la fuerza política de los municipios permanecía algo limitada. Por un lado, sus tesorerías carecían de fondos,

\footnotetext{
11 Las colonias agrícolas establecidas fueron las de Sonora, Herradura, Benito Juá rez y Zaragoza. Como consecuencia de estas medidas, la población del Valle de Mexica li aumentó de poco más de 1,000 habitantes en 1910 a más de 10,000 en 1920. Alrededor de la mitad de este total eran mexicanos, mientras que la otra mitad provenía de otros países: 4,000 eran chinos y el resto estadounidenses. Carr (1917:65); The Calexico Chronicle (1919); The San Diego Union (1920); Cantú (1956:65-66); Rolland (1993:125132,153-155); Keith (1951:46-49).

12 El Camino Nacional, que en su primer tramo conectaba a Mexicali con Tecate, fue extendido posteriormente a Ensenada y Tijuana, aś como entre estos dos últimos puntos. Esta red carretera también se conocía popularmente como el Camino Militar, por haber sido trazada y construida por los ingenieros militares adscritos al gobiemo de Cantú, y para el rápido desplegamiento de tropa en caso de un ataque por parte del gobierno de Carranza.Carr (1917:65). Walther (1986:180). Se contemplaba la extensión del camino en el futuro a lo largo de toda la península. Walter F. Boyle, el cónsul estadounidense en Mexicali, al Departamento de Estado, 25 de agosto de 1920, en U.S. Department of State, Record Group 59, file 812.00, Records of the Department of State Relating to the Internal Affairs of Mexico, 1910-1929, Microcopy 274, National Archives and Records Service, Washington, D.C., documento núm. 24495; Rolland (1993:155-156, 160); Carr (1917: 33, 67); The San Diego Union (1920); Stowell (1921:27-30); Cantú (1956:66); González (1991:260-262); Anguiano (1995:49-56).
} 
dado que la administración distrital absorbía la mayor parte de los impuestos. Por otro lado, el gobernador tenía en sus manos las riendas del poder. Varios ciudadanos locales destacados, tales como Da vid Zárate, Enrique B. Cota y Tomás Ojeda, solicitaron al gobierno federal quese respetara la autonomía municipal y se instalara un gobierno civil en el distrito. También solicitaron que se nombrara a un nativo de la región como gobernador. Aunque existían elecciones municipales, se convirtieron en meros plebiscitos en los cuales $s$ aprobaban los candidatos favorecidos por el gobernador (Samaniego, 1994:75-76;Martínez,1991:553). En marzo de 1917 se creó la municipalidad de Tecate, pero su Ayuntamiento también seguía dominado por el gobernador. Debido a pugnas internas, a principios de 1923 fue disuelto por el gobernador José Inocente Lugo, y se convirtió en una sección municipal deTijuana al ser establecida como municipalidad a mediados de octubre de 1925 (PODNBC, 11 de marzo, 10 de junio de 1917; Walther, 1985:88-103).

A pesar de la debilidad del poder político al nivel local, fue durante la administración de Cantú cuando surgió la manifestación formal más antigua de un movimiento regional en favor de la crea ción de un estado en la región. Esto ocurrió el 26 de enero de 1917, cuando los ayuntamientos de San José del Cabo, Mulegé, San Antonio, Todos Santos y Santa Rosalía solicitaron al Congreso de la Unión que se erigiera como entidad toda la región peninsular. Aunque la petición no dio resultado, dado que el territorio no contaba con los requisitos constitucionales para su conversión en estado, se había establecido un precedente importante en este sentido. ${ }^{13}$

\section{El surgimiento de los primeros Comités Pro-Estado}

El auge derivado de los impuestos sobre la venta de licores y otras formas de diversión a raíz de la Ley Seca en Estados Unidos (1919-

\footnotetext{
13 Pérez Ramírez, "Diputados únicos hasta la creación del Estado," en Archivo His tórico del Estado de Baja California,Mexicali, B.C., Colección Pedro F. Pérez y Ramírez (de aquí en adelante citado como AHEBC/ CP), documento no. 139 (3 de diciembre de 1978); Caballero (1953:10); Sández (1979:134); Dueñas (1988:69); Aguirre (1990:366).
} 
1934) sentó las bases para el establecimiento de una economía regional que, con el tiempo, permitiría a la administración territorial hacerse cargo de los gastos públicos y reducir las aportaciones federales.

El desarrollo de una fuerte base administrativa y económica para la región constituyó una de las prioridades del régimen del gobernador Abelardo L. Rodríguez (1923-1929) (Rodríguez, 1993:122140, 211-249; Rodríguez, 1962:127-132).

Una de las primeras reformas de Rodríguez consistió en prohibir que los empleados públicos, tanto los del gobierno federal como los de la región, residieran en el otro lado de la línea, en Calexico 0 en cualquier otra parte de Estados Unidos. Se dio a aquellas personas afectadas por esta disposición un plazo razonable para fijar sus residencias en territorio nacional. También les proporcionaron préstamos en efectivo para la construcción de casas-habitación; al mismo tiempo se consiguió, por medio de la Secretaría de Hacienda, la libre importación de materiales para la construcción de viviendas y obras públicas (Rodríguez, 1993:55). Esta reforma apoyó la creación de un núcleo administrativo propio en Baja California Norte, que posteriormente serviría para el establecimiento de un aparato gubernamental estatal.

El auge económico resultante de la Ley Seca permitió el establecimiento de un núcleo industrial, así como de fuentes de trabajo en el distrito. Se fundaron varias compañías despepitadoras de algodón, que se dedicaban a producir productos derivados de las semillas, ta les como el jabón, el aceite, la borra, la harinolina y la cascarilla. Otras industrias que tuvieron amplio desarrollo en el distrito en este periodo fueron la harinera y la cervecera. También fue notable la fábrica de æroplanos que se estableció en Tijuana en noviembre de 1927.14

14 La fábrica de aviones posteriormente fue trasladada a México para continuar la construcción de aparatos para usos militares. Correspondencia relacionada con la propuesta de Abelardo L. Rodríguez, fechada en 1928, para la compra de 20 aviones y que sugiere el establecimiento de una fábrica de aeroplanos. AGN, Fondo:"Obregón-Calles", exp. 713-B-19; en el IIH/ UABC, caja 8, exp. 7; Pérez (1956: 133-137); Rodríguez (1962:136-137); Estrada (1983:391-394). 
La nueva prosperidad también permitió que los ayuntamientos realizaran obras y servicios públicos de distinta índole. ${ }^{15} \mathrm{El}$ gobierno del distrito utilizó una porción de los ingresos para la compra de terrenos y su distribución entre pequeños agricultores, muchos de los cuales eran mexicanos repatriados de Estados Unidos, o para la formación de cooperativas agrícolas A estos colonos les fue proporcionado crédito, así como facilidades para la adquisición de maquinaria y otras necesidades (Rodríguez, 1993:122-140/ 1962:127132; Pérez, 1956:116-126).

La autonomía política a nivel local experimentó un revés durante este periodo al ser derogados los municipios libres En 1925 el gobernador Rodríguez declaró disuelto el Ayuntamiento de Me xicali y estableció en su lugar un concejo para administrar el municipio; ${ }^{16}$ también hizo lo mismo en el caso de Tijuana, que tuvo efecto a partir del 1 de enero de 1926. En 1927, el Congreso nacional aprobó la Ley Orgánica y Reglamento de Elecciones Municipales del Distrito y de los Territorios Federales; el Artículo 113 suprimió los municipios en los territorios y estableció en su lugar delegaciones y subdelegaciones (Pérez Ramírez, 1975; Walther, 1986:197/ 1991:107;Aguirre, 1990:272-276, 367).

En lugar de coartar la vida política en la región, la derogación de los municipios tuvo el efecto de estimular las actividades de este tipo así como de promover la organización de grupos políticos.

Como parte de este incremento de la actividad política en la región, en 1929 se formó el primer Comité Pro-Estado de Baja California, integrado por una mezcla de profesionistas, periodistas y personas de otras ocupaciones de la región. Su cuadro dirigente estaba compuesto por las siguientes personas: Miguel Santacruz, pre-

\footnotetext{
15 Una descripción detallada de las obras públicas realizadas durante el periodo de 1924-1927, se encuentra en Rodríguez (1962:211-249). Véase también Pérez (1956:126-128).

16 Decreto del gobernador Abelardo L. Rodríguez referente a la desintegración del Ayuntamiento de Mexicali y el nombramiento de un Concejo Municipal Provisional, 8 de abril de 1925. AGN, "Obregón-Calles",exp. 428-M-33; en IIH/ UABC, caja 4, exp. 3.
} 
sidente; Ricardo Romandía, secretario; Alfonso Cota y Luis Vargas Piñeira,tesorero y subtesorero, respectivamente; y LuisAmador, $H$. Sotelo y F. Márquez, vocales Aunque las actividades de este grupo no lograron tener resultados inmediatos, por lo menos sus representantes lograron entrevistarse en la capital con el Secretario de Gobernación, Carlos Riva Palacio, quien prometió prestar su influencia en persecución de sus fines (Silva, 1951:65; Sández, 1979:135; Dueñas, 1988:70;Aguirre, 1990:367).

El 30 de diciembre de 1930, el presidente Pascual Ortiz Rubio decretó la creación del Territorio Norte de la Baja California, junto con el Territorio Sur de la Baja California y el Territorio de Quinta na Roo. El Territorio Norte de la Baja California quedaba subdividido en tres delegaciones - Mexicali, Tijuana y Ensenada- , cuyas cabeceras se ubicaban en los pueblos del mismo nombre. ${ }^{17}$ La elevación del Distrito Norte a la categoría deTerritorio constituyó un retraso de varias décadas. Como ya se indicó, a partir de 1888 la península quedó dividida en dos subregiones distintas que, a pesar de la considerable extensión territorial de cada una, habían sido denominadas como "Distritos". En 1920, el presidente Adolfo de la Huerta había enviado al Congreso un proyecto de reformas a la constitución nacional, con la recomendación de que se asignara la categoría de territorios a estos "distritos". Aunque se aprobó el cambio, el asunto quedó archivado y nunca adquirió la forma de disposición legal (Ferrer de Mendiolea, 1956:761; Dueñas, 1988:69).

Durante la administración de Ortiz Rubio, el gobierno federal reiteró su política sobre la "mexicanización" del territorio. En 1931, Juan Andreu Almazán, el Secretario de Comunicaciones y Obras Públicas, declaró "ponerse en contra de tales obras (la cons trucción de caminos en Baja California con objeto de ligarla con $\mathrm{E}$ resto de la república) sería un desatendimiento absoluto del peligro

17 El decreto no se publicó oficialmente hasta el 7 de febrero de 1931. Véase el Dia rio Oficial de la Federación (1931); Walther (1986:210). 
verdadero e inmediato de una nueva mutilación de la patria."18 El objetivo de esta política consistió en terminar con el problema del aislamiento de la región, promover su poblamiento con mexicanos y prohibir la adquisición de terrenos por extranjeros. Se elaboraron propuestas fiscales para el desarrollo de las comunicaciones, la coIonización y turismo de la zona. Al considerar los fuertes nexos económicos entre la península y Estados Unidos, así como la fuerte influencia estadounidense en la región, también se consideraba preciso promover el sentimiento cultural y nacional entre los baja californianos. ${ }^{19}$

Muchos bajacalifornianos se sintieron ofendidos por la llamada política de "mexicanización". Ya se consideraban como mexicanos y no "terrinorteños", término utilizado por los políticos del centro para referirse a los ciudadanos de la región. Se sentían incomprendidos e ignorados por los ciudadanos del resto del país (Pérez y Ra mírez, documento no. 54, 1972). Desde principios del siglo pasa do, los mexicanos del interior de la República habían criticado a los fronterizos por su supuesto gusto de aprender el idioma inglés y su tendencia a adoptar hábitos de consumo, costumbres y estilos de vestir estadounidenses. En los libros y otras publicaciones en Méxi-

\footnotetext{
18 Informe que el C. General de División, Juan Andreu Almazán,Secretario de Comunicaciones y Obras Públicas, rinde al $C$. Presidente de la República, con relación a su viaje por el noroeste de México. México, julio de 1930, en el Archivo Histórico "Gena ro Estrada", Secretaría de Relaciones Exteriores, México, D.F., exp. 30-26-54.Almazán hizo esta declaración después de haber regresado de su recorrido por el Distrito Norte el año anterior. Véase también Chamberlin (1949:52); Salazar (1980: 362).

19 Ing Aurelio Leal Treviño, del Distrito Federal, al Secretario de Gobernación,13 de enero de 1931; Cristófero Ibáñez, secretario particular del presidente, al Secretario de Gobernación, 16 de febrero de 1931. AGN, "Dirección General de Gobierno"; en IIH/ UABC, Serie 2.000 (30) 11, caja 57, exp. 22;"Proyectos para la mexicanización de Ba ja California." AGN, "Dirección General de Gobierno", en IIH/ UABC, Serie 2.000 (30) 11 , caja 9, exp. 38. Algunos bajacalifornianos concordaron hasta cierto punto con estas opiniones. Véase las propuestas sometidas a la consideración del gobierno federal a lo largo del año de 1938 por parte de Luis Urbina, vecino de Ensenada, referentes a la construcción de una presa en San Quintín y caminos en la región, como medidas para contra rrestar la influencia estadounidense en la zona. AGN, "Pascual Ortiz Rubio", Vol. 1930, exp. 260/ 10376; en Iн/ UABC, caja 2, exp. 4.
} 
co y el extranjero durante las décadas de 1920 y 1930, casi siempre se desprestigiaban a la región del norte y sus habitantes. 20

A los dos primeros gobernantes del nuevamente constituido territorio - CarlosTrejo y Lerdo de Tejada y Arturo Bernal- les fue encargada la tarea de implementar la política de "mexicanización" en la región (Pérez Ramírez, documento no. 14, 1968). Sus esfuerzos fueron obstaculizados por la depresión económica que se había iniciado en 1929 y que provocó un aumento dramático en el número de mexicanos sin trabajo en Baja California y otras regiones de la frontera.21 Como medida de emergencia, el gobernador Trejo prometió a los sindicatos bajacalifornianos la repartición inmediata de alimentos. Inició varias reformas administrativas, tales como un reajuste en los salarios, la supresión de plazas no consideradas indispensables, así como el establecimiento de un control en las compras y los gastos públicos. Con el propósito de reducir el número de desempleados, se solicitó al gobierno federal una partida presupuestal para poder llevar a cabo obras públicas en las áreas urbanas 22 Arturo Bernal, el sucesor de Trejo a partir del 26 de agosto de 1931, intentó fomentar las inversiones en la región, con la idea de crear fuentes de empleo. A pesar de la necesidad de estimular el crecimiento

\footnotetext{
20 Pérez Ramírez (documento no. 39,1971). Esta actitud tenía ráces muy hondas en el pasado; la cultura de la frontera norte siempre había sido considerada por muchas personas, tanto mexicanos como extranjeros, de alguna manera distinta a la de otras regiones del país En 1828, por ejemplo, un escritor mexicano llamado José María Sánchez, al comentar sobre los efectos nocivos de la proximidad a Estados Unidos para los residentes del pueblo de Nacogdoches en la provincia deTexas, declaró que, "Acostumbrados al continuo comercio con los americanos del Norte, han imitado sus costumbres y así es que se puede decir, con verdad, que (ellos) no son mexicanos más que en el na cimiento, pues aún el idioma castellano lo hablan con bastante ignorancia de él". Cita do en Weber (1988:279).

21 A pesar de la gravedad de la situación, no ocurrió ningún levantamiento en armas entre los pobladores de la región; hubo, empero, casos de saqueo de algunas tiendas de abarrotes. Navejas (1993:37); Sánchez (1990:49).

22 Cientos de las personas deportadas de Estados Unidos fueron enviadas al sur de México, donde existían más posibilidades para asimilarlas a la economía Navejas (1993:37).
} 
económico de la zona, rehusaba, sin embargo, permitir la venta de tierras a compradores extranjeros. 23

A finales de agosto de 1933, Abelardo L. Rodríguez, como presidente de la República, proporcionó algún alivio para los municipios a aprobar la Ley de Perímetros Libres, que estableció aTijuana y Ensenada como distritos con la franquicia de introducir mercancías extranjeras sin pagar derechos. 24 La aprobación de esta ley, que

23 Pérez Ramírez (documento no. 17,1967). Hubo un aspecto en el cual la Gran Depresión sirvió para estimular el poblamiento del territorio y, de esta manera, su "mexicanización." Con el apoyo de poco más de cien mil pesos proporcionados por el Comité Nacional de Repatriación, se establecieron colonias de mexicanos repatriados de Estados Unidos - muchos de los cuales habían sido deportados forzosamente- , en Mexicali,Tijuana, el Valle de San Q uintín, el Valle de las Palmas, y en la antigua colonia Carlos Pacheco, sobre terrenos que ya ocupaban japoneses nacionalizados como mexicanos. Excésior, 24 de octubre de 1931; El Universal, 5 de marzo, 10 de octubre de 1931, 2 marzo de 1933, 18 de septiembre de 1934, 29 de septiembre de 1936, 3, 8 de marzo de 1937; Starr-Hunt (1933:20); Salvador España, del Club Nacionalista Pro-Baja Ca lifornia, Los Angeles,Cal., al Gral. Abelardo Rodríguez, 7 junio de 1934. AGN, "Abelardo L. Rodríguez", exp. 181/ 14; en IIH/ UABC, caja 1, exp. 80; Diario Ofidal, 12 de marzo de 1935; Carta de Cárdenas a la Confederación de Uniones de Campesinos y Obreros Mexicanos del Estado de California, 14 de julio de 1939, reproducida en Bustamante (1986:18-19). Aun cuando, además de estos inmigrantes, llegaron otros en la forma de refugiados republicanos españoles durante la segunda mitad de la década de los treinta, el número de estos colonizadores era reducido. El Universal, 12 de agosto de 1939; $\mathbf{H}$. de León-Portilla (1989:147-148, 157).

24 Solicitud del Gral. Agustín Olachea,gobernador del Territorio Norte de Baja Ca lifornia, al Secretario de Gobernación, para que se establezcan zonas libres en Tijuana y Ensenada, 31 de julio de 1933. AGN, Fondo: "Dirección General de Gobierno", Serie 2.233(30)4, caja 9, exp. 15; en IIH/ UABC, caja 15, exp. 10; Irigoyen (1935:379-382). Rodríguez contemplaba que la creación de los perímetros libres estimularía el desarrollo de la agricultura en la entidad, por medio de la importación de maquinaria agrícola.Opinaba que también permitiría una expansión del comercio y de las ventas, que re sultaría en una economía mucho más diversificada que una que estaba basada esencialmente en los bares y casinos. Propuestas de Julián Velarde al presidente Rodríguez, 3 de febrero de 1934, referentes a la construcción de obras de irrigación en Santo Domingo, B.C., y para aprovechar el desarrollo de los perímetros libres. AGN, Fondo: "Abelardo L. Rodríguez",exp. 106/ 32; en IIH/ UABC, caja 1, exp. 30. Queja de la Cámara Nacional de Comercio de Tijuana al Secretario de Comunicaciones y Obras Públicas por las acusaciones de la compañía Ferrocarril de Tijuana y Tecate a causa del establecimiento del perímetro libre, 23 de mayo de 1934. AGN, Fondo: "Abelardo L. Rodríguez",exp. 571.4/ 11; en IIH/ UABC, caja 6, exp. 45. 
posteriormente fue ampliada para incluir a Mexicali, Tecate y San Luis Río Colorado, Sonora, fue motivada en gran parte por el sector comercial bajacaliforniano, dado que permitió el desarrollo en la región de una economía mucho más diversificada que una basada esencialmente en los bares y casinos, que había prevalecido en la zona hasta aquel entonces. 25

Aunque la crisis económica provocó un estancamiento en el desarrollo de la región y la conformación de una población estable, el malestar entre la gente fue uno de los factores principales que condujo, durante los años de 1931 a 1935, al surgimiento de otros movimientos pro-Estado.

En junio de 1931, el año en que el gobierno federal elevó los Distritos Norte y Sur a la categoría deTerritorios nacionales, se fundó el Comité Pro-Estado Libre y Soberano de Baja California, integrado por representantes de una variedad de profesiones y oficios: empresarios, comerciantes, periodistas, dirigentes laborales, estudiantes, etcétera. Sus miembros más destacados eran Braulio Maldonado Sández, Rafael Osuna, Guillermo Caballero, Francisco Dueñas Montes, Angel Martínez y Domingo Carballo.

Dos de sus miembros en particular - Braulio Maldonado y Francisco Dueñas Montes- desarrollarían importantes papeles en el movimiento pro-Estado a lo largo de sus primeras tres décadas y en la formación del primer Congreso Constituyente de 1952-1953. Sus

\footnotetext{
25 Estudio presentado por delegados de las Secretarías de Hacienda y Economía Nacional sobre las repercusiones negativas y positivas en las actividades económicas de Tijuana y Ensenada, por el establecimiento del perímetro libre, 27 de marzo de 1935. AGN, Fondo: "Lázaro Cárdenas", exp. 564.1/ 26; en IIH/ UABC, caja 16, exp. 11; Periódico Oficial de Territorio de Baja California, 30 de junio de 1937; Propuesta para el establecimiento de un perímetro libre en Mexicali, y una zona libre en el Territorio Norte de Baja Ca lifornia, s.f. AGN, Fondo:"Dirección General de Gobierno", Serie 2.382(30)24554 Tomo I, caja 70, exp. 19/7; en IIH/ UABC, caja 26, exp. 59. Cárdenas al Secretario de $\mathrm{Ha}$ cienda y Crédito Público para que se extienda la zona libre a toda la península, 14 de julio de 1939. AGN, Fondo:"Lázaro Cárdenas", exp. 564.1/ 26; en IIH/ UABC, caja 16, exp. 3. Ampliación de la vigencia del régimen de zona libre en el Territorio Norte de Baja Califomia y parte de Sonora, 29 de diciembre de 1945. AGN, Fondo:"Dirección General de Gobierno," Serie 2.300(29)2321; en IIH/ UABC, caja 15, exp. 36; Irigoyen (1943:138-139).
} 
respectivas carreras son ilustrativas del grupo o clase profesional de donde provinieron varios de los integrantes de los Comités Pro-Estado.

El primero de estos hombres, Braulio Maldonado, nació en San José del Cabo, del entonces Distrito Sur de la Baja California, en 1903. Después de vagar algún tiempo por Estados Unidos y de servir como soldado en el ejército federal mexicano, ingresó, a media dos de la década de los veinte, a la Facultad de Derecho de la UnAM, en donde se tituló como abogado en 1929. A partir de 1930, al integrarse al gobierno del general Agustín Olachea Avilés, se dedicó con tesón a la lucha para mejorar las condiciones laborales y socia les de los obreros y campesinos de la península Sirvió como diputado federal en varias ocasiones, primero por el Territorio Sur de Ba ja California y después por el Territorio Norte. Participó en la elaboración de la Ley Federal deTrabajo, cuya primera versión había sido promulgada en 1931, y también se dedicó a organizar la Coalición Nacional Revolucionaria. Su carrera política vertiginosa culminaría en su elección, al convertirse el Territorio Norte en estado, como el primer gobernador constitucional de la entidad (Maldonado, 1986:10-65).

Francisco Dueñas Montes, por su parte, había nacido en Mexica li en 1908. Entre 1924 y 1929, estudió pedagogía en la Escuela $\mathrm{Na}$ cional de Maestros en la ciudad de México, después se dedicó otros cuatro años (1931-1935) a estudiar medicina en la Universidad Nacional Autónoma de México. Después de egresar como pasante, regresó a su ciudad natal para ejercer la carrera de médico. Poco después de su llegada, fue designado subdirector del Hospital Civil Abelardo Rodríguez, fundado en 1927. A lo largo de su carrera como médico, Dueñas Montes desempeñó una actividad notable en los campos de la educación y la medicina de la región norte de Ba ja California. Fue fundador de varias escuelas en la entidad, entre ellas la Escuela de Enfermería, el Sanatorio Central y el Instituto Regional de Investigaciones Históricas de Baja California "Profesor Pa blo L. Martínez".26

\footnotetext{
26 Entrevista con el Dr. Francisco Dueñas Montes, realizada por el historiador Da vid Piñera Ramírez, del Centro de Investigaciones Históricas de la UABC, Tijuana, en
} 
En 1936, el Comité Pro-Estado envió una petición a la Secretaría de Gobernación y al Congreso de la Unión en la cual se sugirió la conversión de toda la península en un solo estado, en vista del hecho de que ni el Territorio Norte ni el Territorio Sur por sí solos cubrían los requisitos constitucionales para ser elevados a la categoría de estados. 27 Este movimiento fue frustrado por otro grupo de personas que apoyaba el cumplimiento de una propuesta realizada por la Secretaría de Gobernación ante la Cámara de Diputados en 1920 para que la península quedara dividida en dos territorios. Se consideraba que se establecerían dos estados independientes en cuanto estos dos distritos Ilenaran las condiciones legales (Silva, 1951:6566; Dueñas, 1988:7; Walther, 1991:148;Aguirre, 1990:366).

En 1936, también se estableció laAsociación Cívica Pro-Baja Ca lifornia con oficinas en Tijuana, Ensenada y otras poblaciones del territorio. Desde la fecha de su fundación, este grupo, al igual que en el caso del Comité Pro-Estado establecido en 1931, trabajaba incansablemente en favor de la conversión de Baja California en estado. Su fundador y presidente fue el impresor y periodista Alberto Amador Orozco. Nacido en La Paz en 1896, Amador Orozco había sido residente deTijuana desde 1912. A lo largo del periodo territorial,Ama dor Orozco permaneció como uno de los personajes centrales en la vida cívica de la ciudad. Durante la última parte de su vida, fungió como delegado municipal deTijuana (Aguirre, 1975:288; Bonifaz, 1982:265).

También cabe destacar en el caso de este grupo la labor de la profesora Josefina Rendón Parra, quien, aun cuando haya sido única mente miembro y no dirigente de este grupo, hizo una aportación

1981, y publicada en El Mexicano, 26 de julio de 1991; entrevista con el Dr. Francisco Dueñas Montes, realizada en julio de 1991 por la periodista Norma Herrera, publicada en Diario, 29 de julio de 1991, p. 3; Noticia sobre el fallecimiento del Dr. Francisco Dueñas Montes, 25 de mayo de 1997, LaVoz dela Frontera, 26 de mayo de 1997, pp. A-1,3, 10.

27 "Petición que hace el Comité Pro-Estado Libre y Soberano de la Baja California al H. Congreso de la Unión relativa a la erección en estado libre y soberano de losTerritorios de Baja California, en cumplimiento de la fracción II del Artículo 73 de la Constitución General de la República," 24 de septiembre de 1936, en Caballero (1953:11-17); Dueñas (1988:6-7). 
muy notable a esta causa. Nacida en Guanajuato en 1885 , la señorita Rendón Parra llegó aTijuana en 1922 para ejercer la profesión de maestra. Además de dedicar su vida a la docencia y al desarrollo de la educación en Tijuana, escribió varios libros sobre la geografía e historia de la región peninsular. También contribuyó a conservar como tradición local el homenaje a los llamados "defensores" de la región contra los ataques de los rebeldes magonistas en 1911.28

Las condiciones para la realización de la meta de los Comités Pro-Estado serían establecidas durante la administración del presidente Lázaro Cárdenas y el periodo de la Segunda Guerra Mundial. E anhelo del pueblo bajacaliforniano a este respecto se mantendría vivo en gran parte a través del trabajo y empeño de estos diferentes grupos, que seguirían surgiendo a lo largo de toda esta época.

\section{La política de integración nacional cardenista}

Al asumir la presidencia Lázaro Cárdenas en 1934, se implementaron una serie de proyectos, conocidos colectivamente como el Plan Pro-Territorios Federales, con el propósito de integrar los territorios nacionales al resto del país por medio de la colonización y el mejoramiento de los lazos de comunicación. En un discurso que pronunció el 28 de septiembre de 1936, Cárdenas declaró:

Baja California y Quintana Roo han tenido que luchar contra e a aslamiento que se deriva de su situación geográfica especial y de la falta casi absoluta de medios de comunicación hacia el resto de la República [...] debe suscitar un estado de cosas en que ellos cuenten con población mexicana más numerosa, disfruten de protección más efectiva, vivan con el ritmo económico y social de nuestra nacionalidad, mantengan y afirmen las características de la cultura patria, en lugar de seguir luchando desventajosamente, como lo hacen, por neutralizar el efecto de los

28 Salazar ( 1980:348). Es probable que la obra más destacada de la profesora Rendón sea el libro Apuntes históricos deTijuana (1972). 
contrastes que establecen en los lugares inmediatos a comarcas extranjeras de estructura económica más evolucionada [...] , ${ }^{29}$

La primera fase del proyecto para mejorar las comunicaciones entre Baja California y el resto de la República consistió en la ampliación y el mejoramiento del sistema de caminos en la región. El proyecto cardenista se basó en los trabajos iniciados durante el ré gimen de Cantú, y que habían sido continuados por varios de sus sucesores, en particular por los gobernadores Rodríguez y José Inocente Lugo (1922-1923) (Rodríguez, 1993:249-273). Bajo el régimen cardenista, el camino entre Tijuana y Ensenada fue extendido hacia el sur hasta el puerto de San Quintín, a unos 100 kilómetros a sur de Ensenada, que constituyó el primer tramo en la carretera transpeninsular que eventualmente se extendería por toda la península 30 Hacia el este, se amplió el antiguo Camino Nacional hasta San Luis Río Colorado, Sonora. Para mayo de 1940, se había prolongado este camino hasta SantaAna, Sonora, como parte de la proyectada Carretera Federal Número 2 a México. 31

El gran reto del programa cardenista referente al mejoramiento de las comunicaciones en el noroeste consistió en ligar a Baja California con el sistema ferroviario del resto del país. U na vez más, se pudieran aprovechar las obras de construcción realizadas anteriormente, en particular la vía de 17 kilómetros construidos durante la administración del gobernador Lugo como parte de un proyecto iniciado por Cantú con el propósito de conectar el Valle de Mexica

29 Cárdenas del Río (1978:215). Véase también Cárdenas del Río (1936:3-4, 15). Para una discusión interesante de la reacción por parte del público mexicano al discurso, véase El Nacional, México, D.F., 30 de septiembre de 1936.

30 Informe sobre la construcción de la carretera TijuanaEnsenada, B. C., 1932. AGN, Fondo: "Dirección General del Gobierno," Serie 2.300(30)11; en IH/ UABC, caja 157,exp. 21.

31 Este proyecto de camino había sido iniciado durante la primera parte de la década. Correspondencia relativa a la propuesta de Teodoro V. Martínez en 1933 para la construcción de una carretera de Mexicali, B.C., a SantaAna,Sonora. AGN, Fondo:"Abelardo L. Rodríguez", exp. 573.1/ 32; en IIH/ UABC, caja 7, exp. 5. Véase también Salazar (1980:373); Martínez (1991:569). 
li con el puerto de San Felipe y así darle una salida a los mercados mundiales A diferencia de los anteriores proyectos, el objetivo de la construcción cardenista consistió no en reforzar las comunicaciones de la península y el estado de Sonora con Estados Unidos, sino con el resto de México.32 Para 1940, se habían terminado los trabajos entre Fuentes Brotantes (a 64 kilómetros al sureste de Mexicali) y Puerto Peñasco en la costa noroccidental de Sonora. Debido a la suspensión de las obras ordenada por el gobierno del presidente Ma nuel Avila Camacho, así como la escasez de maquinaria y materiales en general provocada por la Segunda Guerra Mundial, el tramo hasta las estaciones de Benjamín Hill y SantaAna en Sonora, se vio obstaculizado hasta 1947 cuando, por medio de un programa de tra bajos intensivos, el proyecto fue concluido en aquel año. ${ }^{33}$

Un tercer medio de comunicaciones se estableció con la inauguración en 1934 por parte de la Compañía Mexicana de Aviación de una ruta aérea que abarcaba las ciudades de Los Angeles, Tijuana y México. Los vuelos de este tipo constituyeron todo un logro al tomar en cuenta la distancia involucrada, aś como el estado incipiente de la aviación en aquella época. El servicio de esta compañía, empero, se suspendió en 1936, y no fue renovado sino algunos años después. 34

32 El proyectado punto terminal de la línea se iba cambiando a través del tiempo. Durante un periodo, por ejemplo, se pensaba construir la línea hasta Santa Isabel ( conocido como Port Isable o Port Otis por los magnates ferroviarios estadounidenses) 0 Puerto Peñasco, entre otros puntos considerados, sobre la litoral sonorense. Pedro Pérez Ramírez, "Apuntes sobre la construcción del Ferrocarril Mexicali-Puerto Peñasco," en AHEBC/ CP, documento no. 27 (abril de 1971); Rodríguez (1993:273); México. Secreta ría de Comunicaciones y Obras Públicas (1948:24-25, 31-32, 66-67); Hendricks (1971:180); Walther (1985:6-7;1986:181-182, 192-193); Barrios (1996:241-242, 253-254).

33 Correspondencia e informes referentes a los proyectos, presupuestos y problemas generados en torno a la construcción del ferrocarril de Mexicali a Puerto Peñasco, 1935-1940. AGN, Fondo: "Lázaro Cárdenas", exp. 545.3/ 63; en IIH/ UABC, Caja 13, exp. 8;Irigoyen (1943:195-204, II:175); México. Secretaría de Comunicaciones y Obras Públicas (1948: 34-50);Barrios (1996:242-243, 255).

34 E cronista Alfonso Salazar Rovirosa indica que el servicio se renovó en 1949, pero Ulises Irigoyen revela que existían vuelos entreTijuana y la capital durante el periodo de la Segunda Guerra Mundial. Irigoyen (1943:168-169); Salazar (1980:365, 369, 
La colonización por mexicanos, aspecto clave del proyecto cardenista para Baja California, exigió un fin al control hegemónico de la tierra del cual hasta entonces habían disfrutado los terratenientes extranjeros. En la realización de este objetivo, el gobierno de Cárdenas pudo aprovecharse de los proyectos colonizadores de los gobiernos anteriores, particularmente los de Cantú y Rodríguez. De acuerdo con el contrato de colonización que la Colorado River Land Company había firmado con la Secretaría deAgricultura y Fomento el 14 de abril de 1936, dicha empresa se había comprometido a vender lo que quedaba de su propiedad del Valle de Mexicali. Si bien se logró satisfacer las necesidades de un número reducido de colonos, aś como formar algunos ejidos, el fracaso del plan de ventas en general, junto con la ocupación forzosa de los terrenos por la llamada Federación Campesina (integrado por cuatro Comités agrarios), dilató el proceso de la redistribución de tierras. Finalmente, en marzo de 1946, el gobierno dispuso que la Nacional Financiera comprara todas las acciones a la antigua compañía y se estableció una nueva empresa, la Compañía Mexicana deTerrenos del Río Colorado, con la responsabilidad de vender sus tierras a colonos mexica nos por cuenta de la nación.

Como consecuencia de esta reforma, tanto la agricultura como en general las actividades económicas de la región experimentaron avances notables. 35 El censo de 1940 mostró el éxito de la meta fe-

382). Existían por lo menos dos companías competidoras, las LíneasAéreas Occidenta les y laVarney Airmail Service, que también realizaban vuelos a la ciudad de México en este tiempo. Arturo Cubillas, Presidente de la compañía Líneas Aéreas Occidentales, al presidente Rodríguez, 23 de febrero de 1934; y correspondencia entre varios particulares con Rodríguez referente al servicio proporcionado por laVarney Airmail Service, 21 de febrero al 7 de marzo de 1934. AGN, Fondo: "Abelardo L. Rodríguez", exp. 574.7/ 4-2; en IIH/ UABC, caja 7, exp. 9. En el ramo de las telecomunicaciones, fue hasta octubre de 1947 cuando se estableció una línea telefónica directa con la ciudad de Mé xico y el resto del país. El Heraldo, Tijuana, B.C., 11 de octubre de 1947.

35 Informes de Dionisio 0 . Mercado relativos a los problemas y avances del proyecto de colonización del Valle de Mexicali, varias fechas,1935-1937. AGN, Fondo:" Lá zaro Cárdenas", exp. 534.3/ 41; en IIH/ UABC, caja 11, exp. 27; Solicitudes de organiza ciones agrícolas y personas de diversos estados de la República y de California, de facilidades para trasladarse al Territorio Norte de la Baja California, varias fechas, 19361939. AGN, Fondo:"Lázaro Cárdenas", exp. 503.11/ 212; en IIH/ UABC, caja 9, exp. 35;In- 
deral a este respecto al mostrar un incremento poblacional de más de 30,500 en el Territorio Norte a lo largo de la década anterior. ${ }^{36}$

Al mismo tiempo, el gobierno cardenista también hizo concesiones a la autonomía regional. Ya se habían hecho algunas anteriormente. El primer caso había ocurrido el 1 de octubre de 1920, cuando el ingeniero Manuel Balarezo, originario del Distrito Sur de Baja California, relevó a Luis M. Salazar en el cargo de gobernador. La segunda ocasión tuvo lugar medio año después, en marzo de 1921, cuando Balarezo fue relevado por Epigmenio Ibarra, quien era de Real del Castillo (Martínez, 1965:770; Calvillo, 1994:52-62). Cárdenas dispuso que el personal asignado a las dependencias de los territorios nacionales pudiera integrarse con personal nombrado de acuerdo con la sugerencia propia del gobernador. Aquellos emplea dos designados a puestos dentro del aparato administrativo local deberían ser nativos de los territorios, o haber radicado en ellos durante por lo menos los cinco años anteriores a su nombramiento. El otorgamiento de un grado mayor de autonomía a la región también ayudó a contrabalancear los efectos de la derogación de los Ayuntamientos durante el gobierno de Rodríguez (Walther, 1991:135).

Las aspiraciones hacia la autonomía regional, junto con el resurgimiento de los comités pro-Estado, volvieron a manifestarse al final del periodo cardenista En 1940, durante la gubernatura del coronel Rodolfo Sánchez Taboada (1937-1944), bajo cuyo régimen se efectuó la política de reforma agraria cardenista, surgió otro Comité Pro-Estado. A diferencia de los movimientos anteriores, la membresía de este nuevo grupo estaba constituida principalmente por funcionarios gubernamentales, ejidatarios, colonos, así como "otros elementos afines y agradecidos al gobierno territorial." Su cuadro

forme del gobernador Rodolfo Sánchez Taboada,gobernador del Territorio de Baja Ca lifornia,sobre la terminación de la entrega de las dotaciones de tierras en el Valle de $\mathrm{Me}$ xicali, 13 agosto de 1937. AGN, Fondo: "Dirección General de Gobierno", Serie 2.382(30)24554 Tomo I, caja 70, exp. 19/ 3; en IIH/ UABC, caja 26, exp. 55; Testimonio del ingeniero Esteban Gómez Pelayo, en Martínez (1991:555-563).

36 Mientras que el censo de 1930 mostró una cifra de 48,327 personas, el de 1940 registró un total de 78,907. Reich (1984:4); INEGI (1994:15). 
directivo estaba integrado por las siguientes personas: Julio Dávila, Enrique Paulín y Arturo M. Escandón (el presidente), por Tijuana; Fausto A. Ramírez y Leandro Rivera, por Mexicali; Jesús Sobarzo, Miguel Santos Torres y Jaime S. Prado, por Ensenada; y Jesús Gracia Fimbres y Fernando Appel Carrillo, por Tecate. Este comité fue reconstituido en 1944, con Escandón como presidente, Carlos M. Kennedy como secretario, José Mapula como tesorero, Manuel Acosta Mesa, como secretario de Organización y Propaganda y Francisco Andrade, Ricardo Gibert y Enrique Palacios como vocales (Pérez Ramírez, 1972; Dueñas, 1988:71; Aguirre, 1990:367-368; Sánchez, 1995:23).

La carrera de uno de sus líderes más notables, Enrique Paulín, constituye un ejemplo del carácter de los miembros más activos de estos grupos pro-Estado durante este periodo. Residente deTijuana desde 1931, Paulín era un destacado dirigente sindical y defensor del establecimiento de la zona libreen la región. También fungió como miembro de varias organizaciones y comisiones fundadas para promover el desarrollo económico y la industrialización de Baja Ca lifornia. Durante la última mitad de la década de los cuarenta, ocupó varios cargos administrativos en el gobierno municipal deTijuana (Aguirre, 1975:299).

Como se verá más adelante, el periodo de la Segunda Guerra Mundial (1939-1945) constituyó desde muchos puntos de vista la piedra angular en el proceso de la conversión de Baja California en estado. El logro principal del trabajo de los Comités Pro-Estado durante los años de guerra reside en el hecho de que dieron expresión popular a esta transformación que estaba ocurriendo en la entidad, al mismo tiempo en que preparó el terreno para el cambio en la estructura gubernamental que vendría después.

\section{El impacto de la Segunda Guerra Mundial}

Aun cuando la Segunda Guerra Mundial constituyó un factor externo en la historia de México, su influencia mostró ser decisiva en el camino hacia la transformación de Baja California en estado. El im- 
pacto de la guerra fue de doble índole; por un lado, se estrecharon todavía más los lazos entre la región y el resto de la república, y por otro, se dio un enorme estímulo al crecimiento de la zona.

El auge en el desarrollo de la región se debió a las demandas impuestas por la guerra en términos de alimentos y otros materiales, junto con la mano de obra mexicana para las industrias bélica y agrícola de Estados Unidos. El desarrollo económico que resultó de la guerra, a su vez, causó que la entidad experimentara un gran aumento poblacional, particularmente en los casos de Tijuana y Mexicali, que se transformaron en centros de servicios, diversión y comercio para miles de soldados estadounidenses apostados en las bases militares de California. 37

Probablemente el aspecto más importante de esta fase de desa rrollo fuera el Programa Bracero, el convenio entre los dos países firmado en agosto de 1942, que estableció la entrada legal de trabaja dores mexicanos a Estados Unidos para suplir una parte importante de la carencia de mano de obra provocada por la guerra. Para Baja California, una consecuencia de este programa fue, para finales del conflicto bélico, un incremento demográfico significativo. Además, los ingresos de los braceros que cruzaron la frontera para trabajar en California - tanto en forma legal como ilegal- dejó una derrama económica importante en el territorio. 38

Al mismo tiempo en que la guerra dio un fuerte estímulo al desarrollo de Baja California, también estableció la base para la zona económica binacional que ligó la franja fronteriza del norte de la península con la economía de la parte sur del estado de California.

37 Entrevistas con Rubén D. Luna, realizada por Guillermo Santana de Bareneche, s.f, y con René Ortiz Campo, realizada por Rigoberto Marín del Campo y Jesús Ortiz Figueroa,1981, en Colección Historia Oral, IIH-UABC; Soto (1990:291-292); Proffitt, III (1994:299).

38 También hubo un programa bracero informal durante la Primera Guerra Mundial, cuando algunos contratistas particulares suministraron a los granjeros california nos trabajadores mexicanos temporales. Scruggs (1960:140-149); Gilmore y Gilmore (1963:265-282);Aguirre (1990:56); Sánchez (1990:51-52). Como ejemplo del desa rrollo demográfico de la entidad, durante la década de 1940-1950 la población de Mexicali se cuadruplicó, mientras que la deTijuana casi se triplicó. Piñeira Ramírez y Ortiz Figueroa (1989). 
El conflicto - mucho más que en el caso de los "perímetros libres"- causó que los fronterizos mexicanos vieran que su futuro económico se situaba en gran parte hacia el norte, a través de la frontera, condición que ha prevalecido hasta la fecha (Proffitt, 1994:299).

A lo largo de la década de los cuarenta, el sector agrícola de Ba ja California experimentó un progreso notable. Esto se debió a va rios factores fundamentales: el reparto ejidal, la agregación continua de nuevos terrenos a la agricultura, la política de la redistribución demográfica - llevar familias enteras de núcleos densamente poblados a regiones que contaban con números reducidos de habitantes- , así como los créditos otorgados a agricultores. Se proporcionaron estímulos a la siembra de cultivos más remunerativos, tales como el algodón, la aceituna, y la uva. El cultivo del algodón en la región de Mexicali experimentó, con la excepción de un breve periodo entre 1945-1946 cuando se abatieron los precios en el merca do mundial, un auge particularmente fuerte durante los años de posguerra (Sánchez, 1990:237-239;Anguiano, 1995:116-123; Irigoyen, 1983:80-81).

De 1940-1950, la población de la entidad se incrementó de 78,907 a 226,967 habitantes, cifra mucho mayor que el número mínimo necesario estipulado por la Constitución $(80,000)$ paraque la región fuera convertida en estado. ${ }^{39} \mathrm{E}$ creciente desarrollo de su economía, particularmente con respecto a la agricultura y la pesca, fue indicativo de que ya contaba con el segundo requisito constitucional, el de tener ingresos propios para cubrir los gastos implícitos

39 INEGI (1994:15). En septiembre de 1943, Juan M. Carrasco Cuéllar, residente del Territorio Norte, había presentado una propuesta al Congreso de la Unión para que la región fuera convertida en estado. Carrasco Cuéllar argumentaba que la población combinada de los Territorios Norte y Sur era más que suficiente para cumplir con la cifra estipulada por la Constitución. También señalaba que aquella región ya contaba con ca si este número de habitantes Propuesta de Juan M. Carrasco Cuéllar para la conversión del Territorio Norte de Baja California en estado, presentada inicialmente el 22 de ma yo de 1943 y sometida a la consideración del Congreso de la Unión el 1 de septiembre del mismo año. AGN, Fondo: "Dirección General de Gobierno", Serie 2.000(30) 27420, caja 58, exp. 18; en IIH/ UABC, caja 9, exp. 69. 
en la organización y el funcionamiento del aparato estatal. No sólo contaba la región con los requisitos formales que se exigían para su conversión en estado, sino que también su situación económica, así como las perspectivas para su futuro desarrollo, eran suficientes pa ra asegurar la continuación de su existencia como estado de la federación.

Esta situación ya era evidente desde mediados de la década de los cuarenta. Durante su campaña presidencial de 1945-1946, Miguel Alemán Valdés había prometido que, al llegar al poder, convertiría el territorio en estado federal (Walther, 1991:148).

El gran empuje dado a la economía regional a raíz de la guerra, junto con el orgullo resultado del desarrollo general de todo el territorio, inyectó un nuevo vigor al movimiento en favor de la autonomía. En 1948, durante la administración del gobernador Alfonso García González (1947-1953), se formó el Consejo Territorial del Comité Pro-Estado (una reorganización de la asociación fundada en 1940) bajo la dirección de Gustavo Aubanel Vallejo, en Tijuana;Francisco Dueñas Montes, en Mexicali; Juan Julio Dunn Legaspy, en Ensenada; y Federico Appel, en Tecate. S bien dentro del liderazgo de este grupo había representantes de una diversidad de sectores socia les, predominaban personas provenientes de las clases profesionales (Silva, 1951:66; Sández, 1979:134-135; Dueñas, 1988:22, 71-72; Aguirre, 1990:368-369).

De este nuevo grupo de líderes del movimiento pro-Estado, quizás la figura más destacada, además del ya mencionado Francisco Dueñas Montes, fue Gustavo Aubanel Vallejo. N acido en Guadalajara en 1904, Aubanal Vallejo llegó a Tijuana en 1931 para ocupar el puesto de director del Hospital Civil y Militar. Durante su carrera como médico-cirujano, desempeñó varios cargos en diferentes instituciones de la ciudad. También fue fundador del primer sanatorio particular deTijuana, aś como miembro fundador de las sociedades médicas regionales organizadas en los años 1932 y 1943, así como del Colegio de Médicos Cirujanos en 1950. Cinco años después de la organización del Comité Pro-Estado de 1948, sería elegido como el primer presidente municipal de Tijuana (Aguirre, 1975:289; Sa lazar, 1980:363; Mercado, 1986:316-317). 
Otro líder notable de este grupo fue el periodista Julio Dunn Legazpy. En 1929, en el períodico El Universal, Dunn Legazpy había redactado un artículo titulado "La verdad sobre la histórica Tijuana," en el cual argumentaba que sus habitantes, quienes en su mayoría $s$ dedicaban a la agricultura y la ganadería, siempre había sido gente honrada y laboriosa. También recordó a sus lectores que eran fervientes patriotas, que lo habían mostrado al defender su pueblo contra los atacantes "filibusteros" dirigidos por Ricardo Flores Magón en 1911 (Romero, 1985:145-146).

El grupo de Mexicali, bajo la dirección de Dueñas Montes, indicó al gobierno federal que su territorio ya superaba en potencialidad económica a los estados de Aguascalientes, Colima, Querétaro, Tlaxcala y Nayarit juntos. También hicieron notar la situación anómala de que, aun cuando Baja California debería contar con municipios libres (de elección popular), a razón de una disposición constitucional del 14 de diciembre de 1940 (Artículo 73, Fracción 6ta, 2da. parte), todavía no se había cumplido con esta prerrogativa 40

El Comité Pro-Estado continuó realizando sus labores con gran ahínco y diligencia durante los años de 1948-1950. En este último año, se formó otro Comité Pro-Estado constituido igualmente por residentes distinguidos de las comunidades del Territorio. A mediados de noviembre de 1951, el presidente Alemán cumplió con la promesa hecha durante su campaña presidencial al enviar al Congreso de la Unión la iniciativa de ley para la conversión del territorio en estado. La iniciativa fue aprobada unánimemente por los diputados y senadores el 31 de diciembre de 1951, y publicada en el Diario Oficial de la Federación el 16 de enero de 1952. Alfonso García González, el gobernador del Territorio Norte, se convirtió en el gobernador provisional del nuevo estado, con el encargo de dirigir la transformación política. 41

\footnotetext{
40 Véase particularmente el artículo "Pro-Estado Libre", sin firma, así como el poema, "A la Baja California (Pro Estado Libre)",Amores Quintana (1949:8).

41 Decreto presentado por el presidente Miguel Alemán Valdés al Congreso de la Unión, 31 de diciembre de 1951, en Martínez (1991:567-571); Diario Oficial, 16 deenero de 1952; Pérez Ramírez, "El Estado de Baja California", en AHEBC/ CP, documento no. 234 (artículo editado en La Voz de la Frontera, 20 de enero de 1985); Caballero (1953:2336); Maldonado (1960:21-24).
} 
El proceso de la construcción política se desarrolló a lo largo de los siguientes dos años y medio. En esta labor, participaron algunos de los líderes de los últimos Comités Pro-Estado, tales como Francisco Dueñas Montes y Gustavo Aubanel.

El 31 de diciembre de 1952, se publicó la convocatoria para la elección de los siete diputados quienes redactarían su Constitución Política. Los diputados constituyentes elegidos en la elección que se llevó a cabo el 29 de marzo de 1953 fueron, sin excepción, candidatos del Partido Revolucionario Institucional (PRI): Celedonio Apodaca, Francisco Dueñas Montes, Aurelio Corrales,Alejandro La madrid, Francisco H. Ruiz, Miguel Calette Anaya y Evaristo Bonifaz Gómez. 42 El texto resultante, si bien no variaba radicalmente del modelo usual para tales documentos, incluía algunas claúsulas que eran innovadoras para aquel tiempo, tales como aquellos artículos que daban protección al patrimonio familiar y el voto a la mujer ( $\mathrm{Pe}$ riódico Oficial de Estado de Baja California, 1953; El Heraldo deTijuana, 1953; González y Grijalva, 1998:175-426).

Poco después de la publicación de la nueva Constitución, se lanzó la convocatoria para las elecciones del gobernador y de la legislatura. Estas elecciones, que se llevaron a cabo el 25 de octubre de 1953, se destacaron por ser las primeras en la historia del país en las cuales votaron las mujeres. En las elecciones para gobernador, Braulio Maldonado, antiguo diputado federal y candidato por parte del PRI, resultó triunfante. La victoria del PRI también fue aplastante en las elecciones congresionales, en las cuales todos sus candidatos derrotaron a los demás contendientes 43

\footnotetext{
42 Véase los recortes de periódico que contienen datos sobre los resultados de la elección, varias fechas, en IIH/ UABC, Fondo:Dirección General de Gobierno, caja 27, exp. 17. Aunque dos de los partidos rivales en la elección, el Partido Acción Nacional (PAN) y la Federación de Partidos del Pueblo (FPP), protestaban a la Comisión Electoral por lo que consideraron como irregularidades, éstas fueron desmentidas por aquel cuerpo. González y Grijalva (1998:32-33).

43 "La mujer en la política de Baja California", El Heraldo de Tijuana, 29 de junio, 25 de agosto de 1953; Alfonso García González al Secretario de Gobernación, 30 de octubre de 1953. AGN, Fondo:Dirección General de Gobierno; en IIH/ UABC, caja 19, exp. 11. Para muchos bajacalifornianos, era significativa la amistad que tenía Maldonado con Adolfo Ruiz Cortines, el entonces presidente de la república, que podría repercutir en la forma de apoyo federal para las obras públicas y otros proyectos. Maldonado había ayu-
} 
El 10 de diciembre de 1953, se publicó en el Periódico Oficial una convocatoria para elegir a los miembros de losAyuntamientos de los cuatro municipios existentes de Mexicali, Tijuana, Ensenada y Teca te, que fueron otorgados con fundamento jurídico propio a razón de la Ley Orgánica Municipal promulgada por el congreso estatal a finales de aquel año. Como resultado de los comicios celebrados en febrero de 1954, Rodolfo Escamilla Soto, Gustavo Aubanel Vallejo, David Ojeda Ochoa y Eufrasio Santana Sandoval fueron electos presidentes municipales de Mexicali, Tijuana, Ensenada y Tecate, respectivamente 44

La última acta en la construcción política del estado ocurrió en la primera semana de julio de 1954, cuando se llevaron a cabo elecciones extraordinarias con el propósito de elegir a los primeros senadores y al segundo diputado federal que correspondía a la eleva ción de la entidad a la categoría de estado, y que lo representarían en el Congreso nacional. Como resultado de estas elecciones, fueron elegidos como senadores Esteban Cantú y Leopoldo Verdugo, así como Aurora Jiménez como diputada federal, así, esa fue la primera vez en México que una mujer ocuparía dicho cargo. 45

\section{Conclusiones}

Las raíces de la autonomía política en Baja California datan esencialmente del Porfiriato. Durante este periodo, la estructura política de

dado a Ruiz Cortines durante su campaña presidencial en Baja California. También ha bía sido amigo íntimo del ex-presidente Miguel Alemán desde la década de los veinte, cuando los dos habían participado, junto con los generales Francisco R. Serrano y Arnulfo R. Gómez, en una revuelta militar contra el gobierno deAlvaro Obregón de 19231924. El candidato también disfrutaba de apoyo entre los sectores campesinos y obreros de la región, con cuyos intereses simpatizaba. Maldonado (1960:13); Maldonado (1986:37, 64); Remembranzas del Prof. Rubén Vizcaíno Valencia, en Murrieta y Hernández (1991:177-178).

44 Para datos sobre esta serie de comicios, véase la documentación que se encuentra en el AGN, Fondo:Dirección General del Gobierno; en IIH/ UABC, caja 17, exp. 52;Ca ja 18, exp. 21.

45 Para documentación en torno a estas elecciones, véase AGN, Fondo: Dirección General de Gobierno; en IIH/ UABC, caja 18, exps. 16,32,33; así como Fondo: Pablo Herrera Carrillo, caja 4, exp. 45. 
la región fue modificándose continuamente, comenzando con la creación de las secciones municipales, que crecieron en número con el tiempo. La vida política ensenadense, en particular, era pujante, con una participación activa por parte de la población civil en las elecciones.

La Revolución Mexicana también tuvo un impacto importante con respecto al desarrollo de la autonomía de la región. A lo largo de la lucha, el Ayuntamiento ensenadense disputaba constantemente con el jefe político sobre sus prerrogativas respectivas y pedía mayor autonomía al gobierno federal. Los primeros movimientos dirigidos hacia la conversión de Baja California en estado también datan de este periodo pero comenzaron a surgir formalmente a finales de la década de los veinte con la aparición de los Comités Pro-Estado. Estos continuaron formándose a lo largo de las siguientes dos décadas. Mientras que se sentaban las bases para una economía autosuficiente y una administración pública propia, los Comités Pro-Estado sirvieron como vehículo imprescindible para canalizar la agitación popular en favor de la autonomía y la conversión de la región en estado.

La política de integración nacional cardenista, que tradicionalmente ha sido vista por los historiadores como una especie de parteaguas en la historia de Baja California en este siglo, reveló la determinación del gobierno federal de llevar a cabo los diversos proyectos de esta índole iniciados por las administraciones anteriores. Aunque la política nacional en este sentido tuvo el efecto de estrechar considerablemente los lazos entre el territorio y el resto de la nación, al mismo tiempo hizo algunas concesiones hacia la autonomía regional.

El periodo que fue particularmente crucial para el proceso de transformación de la región en estado fue el de la Segunda Guerra Mundial, que creó las condiciones necesarias para que esto pudiera ocurrir. La guerra propició que surgiera el movimento Pro-Estado en la región, que llegó a ver cumplida su meta a poco más de seis años de haberse terminado el conflicto. La entidad ya contaba con los requisitos constitucionales, así como con las condiciones necesarias para ser autosuficiente, desde el fin del conflicto en 1945. Se necesitaba, empero, una manifestación por parte de los mismos ba 
jacalifornianos de que estaban preparados y dispuestos para hacerse cargo de sus propios asuntos, deber en el cual los Comités Pro-Esta do de la época de posguerra cumplieron cabalmente.

Recibido en octubre de 1998

Revisado en diciembre de 1998

\section{Bibliografía}

Manuscritos

Archivo General de la Nación, México, D. F. Material fotocopiado de sus diversos ramos que se encuentra guardado en el Instituto de Investigaciones Históricas, Universidad Autónoma de Baja California,Tijuana, B. C.

Archivo Histórico del Estado de Baja California, Mexicali, B.C., Colección Pedro F. Pérez y Ramírez.

Colección de Historia Oral, Instituto de Investigaciones Históricas, Universidad Autónoma de Baja California.

U.S. Department of State, Record Group 59, file 812.00, Records of the Department of State Relating to the Internal Affairs of Mexico, 1910-1929(Microcopy 274), National Archives and Records Service, Washington, D.C.

\section{Libros y artículos}

Aguirre Bernal, C. (1975), Tijuana: su historia, sus hombres, Mexicali, ImprentaTijuana.

(1990), Compendio histórico-biográfico de M exicali, 2 vols., México, Celso Aguirre Bernal. 
Anguiano, M. E. (1995), Agricultura y migración en el Valle de Mexicali, Tijuana, El Colegio de la Frontera Norte.

Barrientes de laTorre, F. (1983), "El trazo original deTijuana", Cala fia, vol. 4, junio.

Bonifaz de Hernández Araico, R. (1982), "Visión económica y social de Ensenada, 1930-1940", en A. Moyano de Guevara y J. Martínez Zepeda (coords.), Visión histórica de Ensenada, Mexicali, Centro de Investigaciones Históricas UNAM-UABC, Universidad Autónoma de Baja California.

Bustamante, J. A. (1986), Historia de la Colonia Libertad, Tijuana, Centro de Estudios Fronterizos del Norte de México.

Caballero Sosa, G. (1953), Pro- Estado de Baja California, Tijuana, s.e.

Calvillo Velasco, M.(1994), Gobiernos diviles de Distrito N orte de la Baja Ca lifornia,1920-1923, México, Instituto Nacional de Estudios Históricos de la Revolución Mexicana.

Cantú Jiménez, E. (1956), “Apuntes Históricos de Baja California”, en Pasajes históricos de la Revolución en $A$ Distrito N orte de Baja California, Tijuana, Instituto de Investigaciones Históricas del Estado de Baja California.

Cárdenas del Río, L. (1978), Palabras y documentos públicos de Lázaro Cárde nas, 1928-1940, 3 vols., México, Siglo xxI.

(1936), El problema de los territorios federales, un llamamiento al pa triotismo y al sentido de responsabilidad de pueblo mexicano, México, Talleres Gráficos de la Nación.

Carr, H. (1917), "The Kingdom of Cantú: Why Lower California is an Oasis of Perfect Peace in Bloody Mexico", Sunset Magazine, vol. 38, abril. 
Comité de Planeación para el Desarrollo del Estado de Baja California, Estadísticas generales de Baja California, Mexicali, COPLADE, 1983.

Chamberlin, E. K. (1949), United States Interests in Lower California, tesis doctoral, Los Angeles, California, University of California.

(1951), "Mexican Colonization versus American Interests in Lower California". Padific Historical Review, vol. 20, febrero.

De León-Portilla,A. H. (1989), “La inmigración que trajo la guerra civil española", en D. Piñera Ramírez y J. Ortiz Figueroa (coords.), Historia deTijuana, 2 vols.,Universidad Autónoma de Ba ja California, Centro de Investigaciones Históricas, UNAM-UABC.

Dueñas Montes, F. (1988), Historia del constituyente en e Estado de Baja Ca lifornia, Mexicali, Instituto de Historia del Estado de Baja California.

Dunn Legaspy, J. J. (1956), “Etapa cantuista en plena revolución, durante cinco años en la Baja California", en Pasajes históricos de la Re volución en el Distrito Norte de Baja California, Tijuana, Instituto de Investigaciones Históricas del Estado de Baja California.

Estrada Lozano, J. A. (1983), “La fábrica de aviones en Tijuana”, en D. Piñera Ramírez (coord.), Panorama histórico de Baja California, Tijuana, B. C., Centro de Investigaciones Históricas, UNAM-UABC.

Ferrer de Mendiolea, G. (1956), "La creación del Estado de Baja Ca lifornia", en Memoria de Primer Congreso de Historia Regional, 2 vols., Mexicali, B.C., Dirección General de Acción Cívica y Cultural, Gobierno del Estado de Baja California.

Francisco Bazán, C. (1989), "Evolución política”, en D. Piñera Ra mírez y J. Ortiz Figueroa (coords.), H istoria deTijuana 2 vols., Universidad Autónoma de Baja California, Centro de Investigaciones Históricas, UNAM-UABC. 
Gilmore, N. R., y G. Gilmore (1963), "The Bracero in California", Pacific Historical Review, vol. 32, agosto.

González Encinas, C. (1991), “Camino Nacional, 1915-1918”, en Mexicali: una historia, 2 vols., Mexicali, Universidad Autónoma de Baja California.

González Oropeza, M., y A. Grijalva (comps) (1998), Digeto constitu cional mexicano: La Constitución Política de Baja California, Mexicali, sEP/ UABC/ Senado de la República.

Hendricks, W. O. (1971), "Port Otis", en San Diego Corral of the Westerners, Brand Book no. 2, San Diego, California, San Diego Corral of theWesterners.

Instituto Nacional de Estadística, Geografía e Informática (1994), Estadísticas históricas de M éxico, México, INEGI.

Irigoyen, U. (1935), El problema económico de las fronteras mexicanas, 2 vols., México, s.e.

(1943), Carretera transpeninsular de la Baja California, 2 vols, México, Editorial América.

Maldonado Sández, B. (1960), Baja California: comentarios políticos, México, CostaAmic.

(1986), Memorias, Mexicali, Talleres Gráficos de Baja California.

Martínez, P. L. (1965), Guía familiar de Baja California, México, Editorial Baja California.

(1991), Historia de Baja California, La Paz, B.C.S, Patronato del Estudiante Sudcaliforniano/Consejo Editorial del Gobierno del Estado de B.C.S. 
Mercado Díaz de León, R. (1986), Los pioneros de la medicina en Tijuana, México, Litográfica Rivera.

Murrieta, M., y A. Hernández ( 1991), Puente M éxi co: la vecindad deTijua na con California, Tijuana, El Colegio de la Frontera Norte.

Navejas Dávila, J. R. (1993), “CarlosTrejo y Lerdo deTejada, primer gobernador del Territorio Norte", Calafia, vol. 7, noviembre.

Ortiz Figueroa, J. (1989), "日 área de Tijuana en el contexto de las divisiones político-administrativas de la península de Baja California", en D. Piñera Ramírez y J. Ortiz Figueroa (coords.), His toria deTijuana, 2 vols.,Tijuana, U niversidad Autónoma de Baja Ca lifornia, Centro de Investigaciones Históricas UNAM-UABC.

Ortiz Figueroa, J. (1989), "El papel de la fuerza rural de Baja California en la estructura política del Porfiriato, 1880-1888", M eyi bó, vol. 3.

Pérez Tejada, G. J. (1956), "General Abelardo Rodríguez”, en Pasajes históricos dela Revolución en el Distrito Norte de Baja California,Tijuana,Instituto de Investigaciones H istóricas del Estado de Baja California.

Proffitt III,T.D. (1994), Tijuana:The History of a M exican M etropolis, San Diego, San Diego State University.

Reich, P. L. (ed) (1984), Statistical Abstract of the United States-M exico Bor derlands, Los Angeles, UCLA Latin American Center Publications.

Rodríguez,A. L. (1962), Autobiografía, México, Novaro Editores. (1993), Memoria administrativa de gobierno de Distrito Norte de la Baja California,1924-1927, 1928; reimpresión, México, SEP/ UABC.

Rolland, M. C. (1993), Informe sobre e Distrito Norte de Baja California, ed. Max Calvillo Velasco, 1919; reimpresión, México, SEP/ UABC. 
Romero Aceves, R. (1985), Baja California: ensayo enciclopédico, México, Costatamic.

Salazar Rovirosa,A. (1980), Historia del Estado de Baja California, 4ta. ed., México, Ediciones Económicas.

Samaniego López, M.A. (1994), "Las luchas políticas en Baja California, 1910-1920", Frontera N orte, vol. 6, enero-junio.

Sánchez Ramírez, 0. (1990), Crónica agrícola del Valle de Mexicali, Mexicali, Universidad Autónoma de Baja California.

(1995), "La instauración del Estado de Baja California", Calafia, vol. 8, diciembre.

Sández Parma, R., (1979), N osotros los bajacaliforniansos, México, Rotopunto Color.

Secretaría de Comunicaciones y Obras Públicas (1948), Dirección General de Construcción de Ferrocarriles, Ferrocarril Sonora-Baja Ca lifornia, México, Secretaría de Comunicaciones y Obras Públicas.

Scruggs, Otey M. (1960), "The Evolution of the Mexican Farm La bor Agreement of 1942", Agricultural History, vol. 34, julio.

Silva Cota, G. (1951), Baja California Norte debeser Estado, tesis de licenciatura en Derecho, México, Universidad Nacional Autónoma de México.

Soto Fuentes, J. (1990), Misión Tijuana, Tijuana, Editorial Acción Cultural Educativa

Starr-Hunt, J. (1933), "The Mexicans Who Went Home", TheAngeles Times, suplemento dominical, 26 de marzo.

Stowell, Jay S. (1921), The Near Side of the M exican Question, New York, George H. Doran Company. 
Taylor Hansen, L. D. (1995), "La revuelta magonista de 1911 en Ba ja California: acontecimiento clave en el desarrollo del sentimiento nacional entre la población peninsular norteña", Frontera Norte, vol. 7, enero-junio.

Valdemar Barrios, M. (1996), Por las rutas del desierto, Hermosillo, Gobierno del Estado de Sonora.

Walther Meade, A. (1982), "La sub-prefectura política de Tijuana", Calafia, vol. 4, diciembre.

(1983), El Partido N orte de Baja California, Mexicali, Universidad Autónoma de Baja California. ciembre.

(1983), "Ensenada, ciudad portuaria", Calafia, vol. 5, di(1984), "Antecedentes y fundación de Tijuana", Calafia, vol. 5, diciembre.

(1985), "San Felipe, enclave en el Mar de Cortés", Calafia, vol. 5, marzo.

(1985), Tecate cuarto municipio, Mexicali, Universidad Autónoma de Baja California.

(1986), El Distrito Norte de Baja California, Mexicali, Universdad Autónoma de Baja California.

(1991), Origen de M exicali, Mexicali, Universidad Autónoma de Baja California.

Weber, D. J. (1988), La frontera de México, 1821-1846: el sudoeste norteame ricano en su época mexicana, México, Fondo de Cultura Económica. 
